\title{
Kinase suppressor of Ras-1 protects intestinal epithelium from cytokine-mediated apoptosis during inflammation
}

\author{
Fang Yan, ${ }^{1}$ Sutha K. John, ${ }^{1}$ Guinn Wilson, ${ }^{1}$ David S. Jones, ${ }^{1}$ M. Kay Washington, ${ }^{2}$ \\ and D. Brent Polk ${ }^{1,3}$ \\ 1Department of Pediatrics, 'Department of Pathology, and ${ }^{3}$ Department of Cell and Developmental Biology, \\ Vanderbilt University School of Medicine, Nashville, Tennessee, USA.
}

\begin{abstract}
TNF plays a pathogenic role in inflammatory bowel diseases (IBDs), which are characterized by altered cytokine production and increased intestinal epithelial cell apoptosis. In vitro studies suggest that kinase suppressor of Ras-1 (KSR1) is an essential regulatory kinase for TNF-stimulated survival pathways in intestinal epithelial cell lines. Here we use a KSR1-deficient mouse model to study the role of KSR1 in regulating intestinal cell fate during cytokine-mediated inflammation. We show that KSR1 and its target signaling pathways are activated in inflamed colon mucosa. Loss of KSR1 increases susceptibility to chronic colitis and TNF-induced apoptosis in the intestinal epithelial cell. Furthermore, disruption of KSR1 expression enhances TNF-induced apoptosis in mouse colon epithelial cells and is associated with a failure to activate antiapoptotic signals including Raf-1/MEK/ERK, NF-KB, and Akt/protein kinase $B$. These effects are reversed by WT, but not kinase-inactive, KSR1. We conclude that KSR1 has an essential protective role in the intestinal epithelial cell during inflammation through activation of cell survival pathways.
\end{abstract}

\section{Introduction}

The homeostatic balance between proliferation and apoptosis is essential for the intestinal epithelium to function as a physiological and structural barrier. Disruption of this balance leads to villus atrophy, epithelial hyperplasia, loss of normal absorptive function, and an increased risk of tumorigenesis. Evidence from a number of different animal and human studies suggests that inflammatory bowel diseases (IBDs), including ulcerative colitis and Crohn disease, are the consequence of abnormal immune responses to pathogenic or nonpathogenic organisms or other environmental stimuli that disrupt this mucosal barrier with increased epithelial apoptosis (1). Altered local cytokine production appears to be critical for inducing pathologically increased rates of epithelial cell turnover in active inflammation (2). Among the cytokines altered in IBD, TNF is a key mediator in the pathogenesis of a number of gastrointestinal diseases with altered mucosal repair, including NSAID enteropathy (1), Helicobacter pylori gastritis (1), celiac disease (3), HIV enteropathy (4), graft-versus-host disease (5), and necrotizing enterocolitis (6).

TNF interacts with 2 receptors, TNFR1 and TNFR2, to initiate distinctive cellular responses. Pathological concentrations of TNF inhibit intestinal epithelial cell wound closure and proliferation through TNFR1 $(7,8)$, whereas activation of TNFR2 by lower TNF concentrations leads to increased intestinal cell proliferation and migration (7-9). Activation of TNFR1 or TNFR2 induces either apoptosis or cell survival, depending upon the balance between anti- and proapoptotic pathways $(10,11)$. TNF-regulated

Nonstandard abbreviations used: DIC, differential interference contrast; ERK, extracellular signal-regulated kinase; I $\mathrm{KB}$, inhibitor of $\kappa \mathrm{B}$; IBD, inflammatory bowel disease; ISOL, in situ oligo ligation; KI, kinase inactive; KSR1, kinase suppressor of Ras-1; MCE, mouse colon epithelial; PKB, protein kinase B.

Conflict of interest: The authors have declared that no conflict of interest exists.

Citation for this article: J. Clin. Invest. 114:1272-1280 (2004).

doi:10.1172/JCI200421022. antiapoptotic pathways include extracellular signal-regulated kinase (ERK)/MAPK (12), NF- $\mathrm{B}$ (12), and Akt/protein kinase B (PKB) (13), whereas proapoptotic TNF-initiated signals include p38 and stress-activated protein kinase (SAPK)/JNK (13-15).

The molecular switches determining the balance between proapoptotic and antiapoptotic TNF signals are not well characterized but include Ras (16) and Raf-1 (17). Additionally, kinase suppressor of Ras-1 (KSR1) regulates TNF-activated antiapoptotic signals in intestinal epithelial cells $(12,13,18)$. KSR1, a 97-kDa protein previously identified as a ceramide-activated prolinedirected Ser/Thr kinase $(19,20)$, is highly conserved from humans to Drosophila and Caenorhabditis elegans $(21,22)$.

Disruption of KSR1 expression or kinase activity blocks activation of ERK1/2 MAPK, NF- $\mathrm{B}$, and Akt/PKB, increasing apoptosis in TNF-treated intestinal cells $(12,13)$. KSR1 functions as both a scaffolding molecule for Raf-1/MEK/ERK and a cell type-dependent kinase for Raf-1 $(18,23,24)$. Interestingly, the KSR1-deficient mouse is grossly normal, but displays defects in ERK kinase signaling, T cell activation, skin development (25), and Ras-dependent tumor formation (26). We therefore hypothesized that KSR1 functions to protect intestinal epithelial cells from cytokine-induced apoptosis in vivo. To test this hypothesis, we examined the regulation of signal transduction pathways and apoptosis in intestinal mucosa of the KSR1-deficient mouse and in a mouse model of IBD.

\section{Results}

Loss of KSR1 enhances TNF-induced apoptosis in colonic epithelial cells in vivo. We have reported that KSR1 regulates TNF-mediated colon cell survival in vitro $(12,13)$. To determine whether KSR1 functions as an antiapoptotic molecule in vivo, we treated $\mathrm{KSR}^{-/-}$mice with TNF and measured apoptosis. After a 4-hour treatment, TNF-induced colon epithelial cell apoptosis was increased up to 3 -fold in the $\mathrm{KSR}^{-1-}$ mice compared with WT mice (Figure 1, A 
A

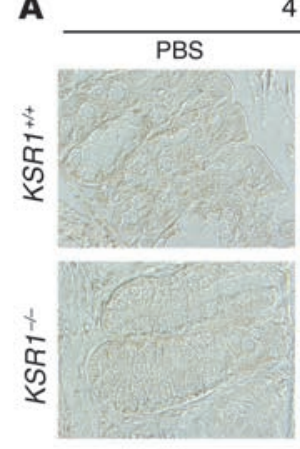

C
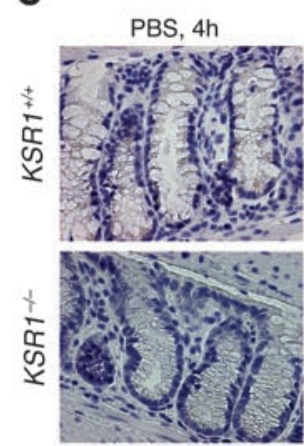

$4 \mathrm{~h}$

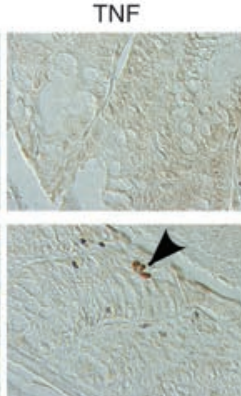

$24 \mathrm{~h}$

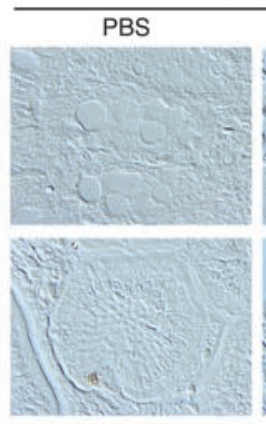

TNF
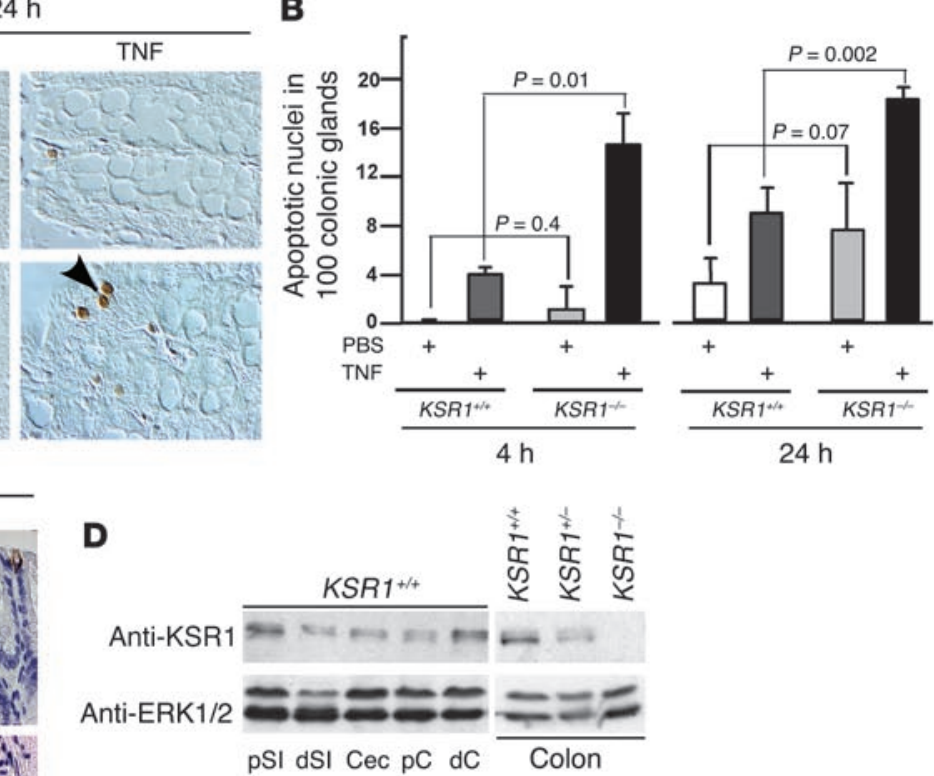

$2 \mathrm{~h}$
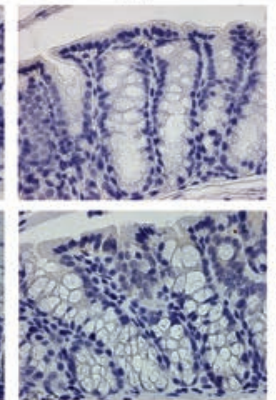

Colon: active caspase-3
E

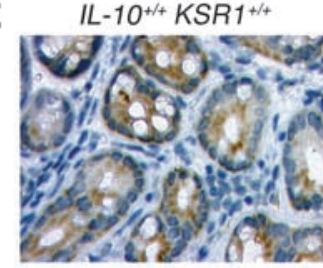

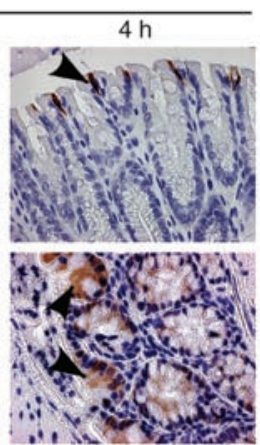

IL-10 $0^{+/-} \mathrm{KSR}^{+/-}$

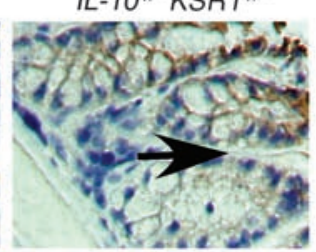

$\mathrm{IL}-10^{-1-} \mathrm{KSR} 1^{+/+}$

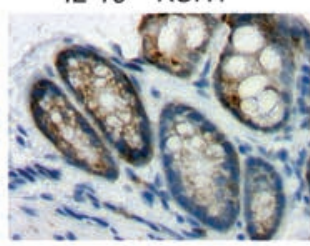

$\mathrm{IL}-10^{+/+} \mathrm{KSR} 1^{-1-}$

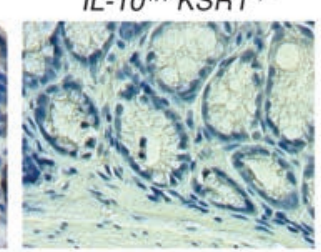

Figure 1

TNF induces apoptosis in KSR1--- mouse colon epithelium in vivo. Mice were injected with TNF or PBS for the indicated times. Paraffin-embedded colon tissues were studied for apoptosis using ISOL staining. (A) Apoptotic nuclei labeled with peroxidase were visualized using DIC microscopy. Arrowheads indicate ISOL-labeled apoptotic nuclei. (B) The number of apoptotic nuclei found per 100 colonic glands. (C) Caspase-3 activity was determined by immunohistochemistry using anti-active caspase-3 antibody. Arrowheads indicate examples of caspase-3-positive cells detected by peroxidase. KSR1 expression in the gastrointestinal tract was determined by Western blot analysis of mucosal lysates (D)

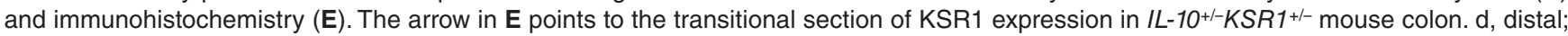
p, proximal; SI, small intestine; Cec, cecum; C, colon. The data shown here are representative of 5 different experiments. Magnification, $\times 40$.

and B). Also, basal apoptosis was increased in $K S R 1^{-/-}$mouse colon epithelial (MCE) cells. To further characterize the apoptotic cells, we performed immunohistochemistry using anti-active caspase-3 antibody. Activated caspase-3 staining was increased in TNF-treated $\mathrm{KSR}^{-/-}$mice within 4 hours, and was limited to the epithelial cell layer (Figure 1C), a pattern identical to the findings with the apoptosis assay. Immunohistochemistry and Western blot analysis showed KSR1 expression throughout the intestine and colon (Figure 1D), with the predominant localization in the epithelial cells (Figure 1E). As expected, no KSR1 was detected in the $K S R 1^{-/-}$ mouse tissue sections. Taken together, these data indicate that TNF regulates intestinal cell fate in vivo through KSR1.

TNF activation of antiapoptotic signals requires KSR1. Because KSR1 mediates TNF-stimulated cell survival pathways in colon cell lines, we studied the in vivo role of KSR1 in these pathways. Protein activation in colon mucosal lysates was determined by Western blot analysis or immunohistochemistry. TNF stimulated activation of ERK1/2
MAPK and Akt/PKB, degradation of inhibitor of $\kappa \mathrm{B}-\alpha(\mathrm{I} \kappa \mathrm{B} \alpha)$, and $\mathrm{NF}-\kappa \mathrm{B}$ nuclear translocation in $\mathrm{KSR}^{+/+}$, but not in $\mathrm{KSR} 1^{-/-}$mice (Figure 2, A and B). TNF increased in vivo expression of the NF- $\kappa \mathrm{B}$ target cIAP2 (12) in colon epithelial cells of WT mice, but not KSR1deficient mice (Figure 2C).

To further determine the regulatory role of KSR1 in TNF signaling, we generated conditionally immortalized MCE cell lines null for KSR1 (KSR1 $1^{-/-}$MCE cells). Compared with young adult mouse colon cells expressing endogenous KSR1 $(12,18,27), \mathrm{KSR}^{1^{-/-}} \mathrm{MCE}$ cells underwent apoptosis upon TNF treatment (Figure 3A), and the percentage of apoptotic cells increased as the period of TNF treatment increased (Figure 3B). The percentage of cells undergoing apoptosis at a given time plateaued after 16 hours, primarily because of overall cell loss (shown by comparison of DAPI-positive nuclei at 24 hours in Figure 3A). TNF failed to stimulate antiapoptotic signals, such as ERK1/2 MAPK, Akt/PKB, and IкB $\alpha$ degradation, in $K S R 1^{-1-}$ MCE cells (Figure 3C). Consistent with our report that 


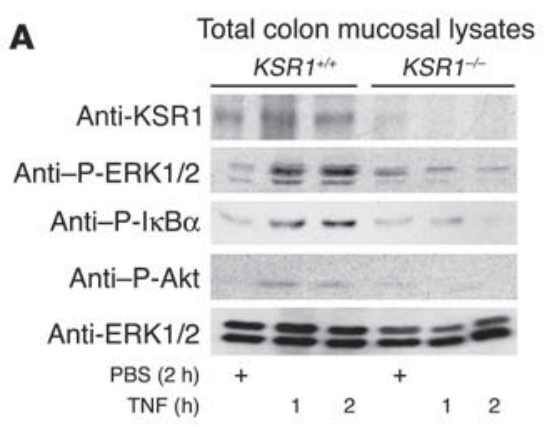

Figure 2

Loss of KSR1 in colon epithelium inhibits TNF-stimulated cell survival signals in vivo. KSR1 $1^{+/+}$or $K S R 1^{-/-}$mice were injected with TNF or PBS for the indicated times. Total colon mucosal lysates were collected for Western blot analysis with anti-KSR1, anti-P-ERK1/2, anti-P-IKB $\alpha$, anti-P-Akt, and anti-ERK1/2 antibodies (A). P, phosphorylation. Paraffin-embedded colon tissues were immunostained with anti-NF-KB p65 subunit (B) or cIAP2 (C), labeled with peroxidase, and visualized using DIC microscopy (magnification, $\times 40$ ). Arrows indicate nuclear localization of NF-kB (B) and clAP2-positive cells $(\mathbf{C})$. Shown is 1 representative of 5 mice in each treatment group.

KSR1 is not required for EGF activation of ERK1/2 MAPK in intestinal epithelial cells (18), EGF initiation of ERK1/2 MAPK activation in $K S R 1^{-/-}$MCE cells was similar to its action in young adult mouse $\mathrm{KSR}^{+/+}$colon cells (Figure 3C). Next, we transiently expressed either WT or kinase inactive (KI) KSR1 in $K S R 1^{-/-}$MCE cells prior to TNF treatment. Expression of WT KSR1, but not KI KSR1, restored TNF activation of ERK1/2 MAPK, Akt/PKB, and degradation of I $\mathrm{B} \alpha$ (Figure 3D). Interestingly, the coimmunoprecipitation assay showed that KI KSR1 and WT KSR1 interacted with similar proteins in MCE cells, including Raf-1 and MEK1/2 (Figure 3E). These data indicate that the KI KSR1 dominant-negative effect on Raf-1/ MEK/ERK activation in mammalian cells is likely due to disruption of kinase activity, not altered Raf-1 or MEK binding capacity. Thus, we interpret these and previous findings to support KSR1 kinase activity as a positive regulator of TNF-initiated antiapoptotic signals determining intestinal epithelial cell fate.

KSR1 is activated in inflamed colon mucosa. IL-10-deficient $\left(I L-10^{-/-}\right)$ mice spontaneously develop IBD by 12 weeks of age with increased TNF and IFN- $\gamma$ production early in the inflammatory process (28). Therefore, we used this model to determine the phosphorylation and activation state of KSR1 during intestinal inflammation. The colon appeared normal in 8-week-old BALB/c IL-10 ${ }^{+/+}$and $I L-10^{-/-}$ mice but showed severe inflammation in 12 -week-old $I L-10^{-/-}$mice (Figure 4A). Inflamed $I L-10^{-/-}$mucosa showed increased activation of ERK1/2, p38 MAPK, Akt/PKB, and IKB $\alpha$ phosphorylation compared with 8-week-old $I L-10^{-/-}$and BALB/c WT mice (Figure 4B). KSR1 and Raf-1 were recovered by immunoprecipitation from mucosal homogenates and the phosphorylation state was determined by anti-phospho-Ser and anti-phospho-Thr antibodies. KSR1 recovered from inflamed $I L-10^{-/-}$colon showed increased Thr but unchanged Ser phosphorylation (Figure 4C). Tyrosine phosphorylation of KSR1 was not detected in inflamed or noninflamed colonic mucosa, consistent with observations in cell culture (18) (data not shown). Interestingly, Raf-1 Thr phosphorylation was increased only in inflamed $I L-10^{-/-}$mucosa (Figure 4D). Similarly, in a 2-step in vitro kinase assay (18), only the KSR1 from inflamed mucosa stimulated Raf-1 Thr phosphorylation and kinase activity toward MEK1 (Figure 4E). Because both KSR1 kinase activity and scaffolding function have been shown to be regulated by phosphorylation $(19,29,30)$, we wondered wheth- er phosphorylation is required for KSR1's kinase activity. KSR1 recovered from inflamed $I L-10^{-/-}$mucosa was divided and either subjected to phosphatase treatment or not, and then tested for kinase activity toward Raf-1 in activating MEK1. Following dephosphorylation, including loss of both phospho-Thr and phospho-Ser, KSR1 was unable to stimulate Raf-1 kinase activity (Figure 4F). These findings indicate that KSR1 Thr phosphorylation level and kinase activity are increased in inflamed mouse mucosa and that kinase activity is regulated by protein phosphorylation.

Loss of KSR1 enhances susceptibility to colon inflammation. As shown above, KSR1 kinase activity is increased during inflammation and the loss of KSR1 enhances TNF-induced apoptosis in colon epithelial cells. Because of increased TNF production during the early phase of colonic inflammation (28), the $I L^{-10^{-/-}}$mouse is ideal to study the role of KSR1 during this process. We crossed $I L-10^{-/-}$mice (expressed on a BALB/c background more than 10 generations) with $\mathrm{KSR}^{-/-}$mice (expressed on a 129/SvEv background more than 10 generations) to test the hypothesis that colon epithelial cell apoptosis would be increased without KSR1. To control for issues of genetic background combinations, we also crossed $I L-10^{-/-}$mice with $129 / \mathrm{SvEv}\left(K S R 1^{+/+}\right)$mice, or BALB/c $\left(\mathrm{IL}-10^{+/+}\right)$mice with $\mathrm{KSR} 1^{-/-}$mice to obtain the same first-generation (F1) background mice with different heterozygous null genes $\left(\mathrm{IL}-10^{+/-} \mathrm{KSR} 1^{+/+}\right.$or $\left.\mathrm{IL}-10^{+/+} \mathrm{KSR} 1^{+/-}\right)$. Surprisingly, the resulting F1 $I L-10^{+/-} \mathrm{KSR} 1^{+/-}$mice developed IBD by 8 weeks of age (Figure 5), characterized by diarrhea and weight loss with an increased histological injury score (Table 1 ). Neither F1 $I L-10^{+/-} \mathrm{KSR}^{+/+}$nor F1 $I L-10^{+/+} K S R 1^{+/-}$mice at the same age showed symptoms of IBD or increased injury scores. Histologically, $I L-10^{+/-} K S R 1^{+/-}$mice showed enterocyte loss, ulceration, crypt inflammation, infiltration of mononuclear and polymorphonuclear cells into lamina propria and mucosal hyperplasia (Figure 5A). Furthermore, apoptosis was significantly increased in $I L-10^{+/-} \mathrm{KSR}^{+/-}$colon compared with either age-matched $I L-10^{+/-} K S R 1^{+/+}$or $I L-10^{+/+} K S R 1^{+/-}$mice (Figure 5 , A and B). These results indicate that KSR1 regulates both antiinflammatory and antiapoptotic signals in the mouse colon.

KSR1 is not required for TNF-regulated endothelin expression in colonic mucosa endothelial cells. Intestinal endothelial cells are a primary target in radiation-induced intestinal injury, with endothelial cell apoptosis preceding epithelial cell death (31). Therefore, we 
A

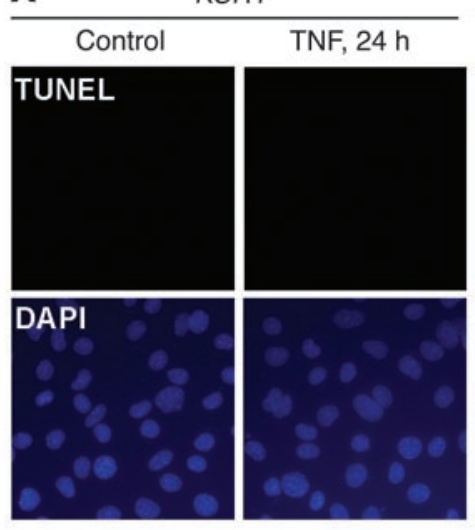

B

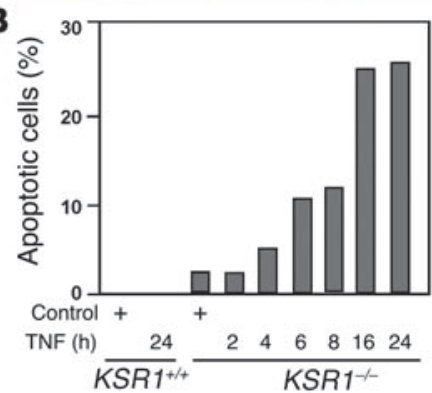

C

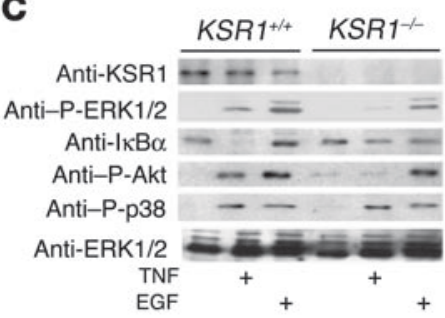

$\mathrm{KSR} 1^{-1-}$

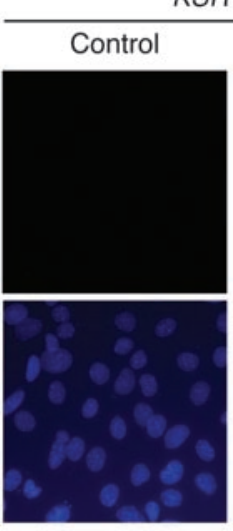

D

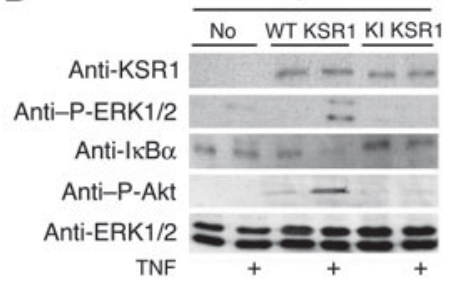

TNF, $24 \mathrm{~h}$
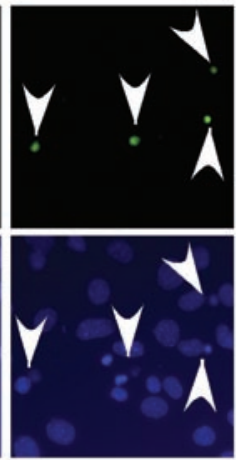

$K S R 1^{-1}$
.

.

\section{Figure 3}

KSR1 regulates TNF-induced survival responses in MCE cell lines. (A) KSR1-l- MCE cells were treated with TNF for 24 hours and fixed for TUNEL and DAPI staining. Apoptotic cell nuclei in TUNEL staining were labeled with FITC (indicated by arrowheads) and visualized using fluorescence microscopy (magnification, $\times 40)$. (B) The percentage of cells undergoing apoptosis following TNF treatment for the indicated times is shown from a representative experiment. KSR1-l- MCE cells $(\mathrm{C})$ or $K S R 1^{-1-}$ MCE cells transiently transfected with WT KSR1 or KI KSR1 (D) were treated with TNF or EGF, and cellular lysates were prepared for Western blot analysis with the indicated antibodies. KSR1 and its coprecipitated proteins from $\mathrm{KSR}^{-/-} \mathrm{MCE}$ cells transiently transfected with vector, WT KSR1 or KI KSR1 were separated by SDS-PAGE and stained with colloidal blue. Raf-1 and MEK bound to KSR1 was determined by Western blot analysis with anti-Raf- 1 or anti-MEK1/2 antibodies (E). Shown are representative data from 3 separate experiments.

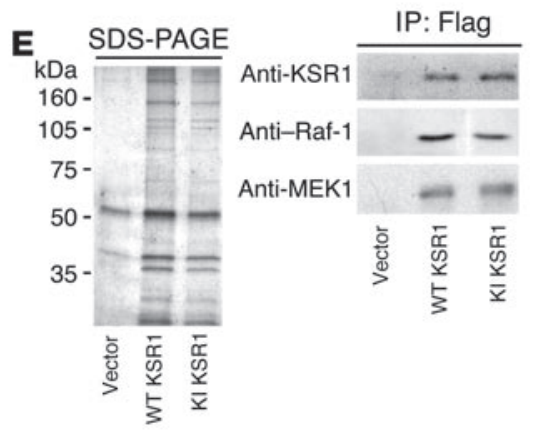

studied the function of KSR1 in endothelial cells in the intestinal mucosa of mice exposed to TNF. In contrast to epithelial cells (see Figure 1C), there was no increase in endothelial cell caspase-3 activation between 1 hour and 24 hours after treatment (Figure 6A). Likewise, there was no increase in endothelial cell apoptosis during the first 24 hours after TNF treatment of either WT or $\mathrm{KSR}^{-{ }^{-/}}$ mice (Figure 6B). Cell culture studies indicate that NF-кB activation is a major antiapoptotic pathway regulated by KSR1 (12), and endothelin is a target of NF-KB signaling in endothelial cells (32). TNF stimulated NF-кB p65 subunit nuclear translocation (data not shown) and endothelin production in mucosal endothelial cells of both WT and $K S R 1^{-/-}$mice (Figure 6C). Thus, disruption of TNF-stimulated NF- $\kappa \mathrm{B}$ activation in $\mathrm{KSR}^{-1-}$ mice does not extend to the intestinal endothelial cell compartment.

\section{Discussion}

A number of disorders of the gastrointestinal tract, such as IBDs, are characterized by elevated TNF production and increased apoptosis that disrupt intestinal homeostasis and the integrity of the protective epithelial monolayer. Therefore, determining the signal transduction pathways regulating intestinal epithelial response to cytokines may identify novel regulatory proteins as therapeutic targets for treatment of IBD or other TNF-mediated intestinal disorders. Our previous studies suggest that KSR1 is a regulatory protein for TNF-mediated cell survival signaling pathways $(12,13$, 18). Our aims here were to determine the in vivo role of KSR 1 in cytokine-induced apoptosis and cell survival signaling in the intestinal epithelium during inflammation. Results in this paper show that in intestinal epithelial cells, KSR1 protects from cytokineinduced apoptotic injury and is required for TNF activation of cell survival signal transduction pathways, including ERK1/2 MAPK, $\mathrm{Akt} / \mathrm{PKB}$, and NF-кB. Thus, loss of KSR1 increases TNF-mediated apoptosis, which may lead to disruption of the defensive function of the epithelial monolayer and to enhanced mucosal inflammation. These studies provide unequivocal evidence that KSR1 activation exerts a protective role in colon mucosal inflammation and the first in vivo indication that mammalian KSR1 functions as a molecular cell survival switch.

KSR1 was originally identified as a ceramide-activated protein kinase prior to its discovery through loss-of-function genetic screening analyses of the biologic actions of Ras signaling pathways in C. elegans and Drosophila $(19,21,22)$. Mechanisms of KSR1 action appear to include both a scaffolding role, linking downstream targets of the Ras/Raf-1/MEK/ERK MAPK signaling 
A
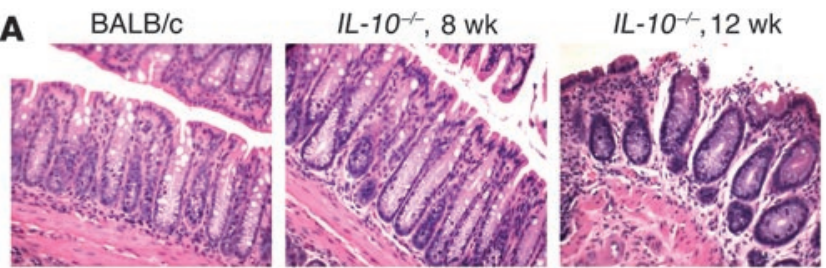

B

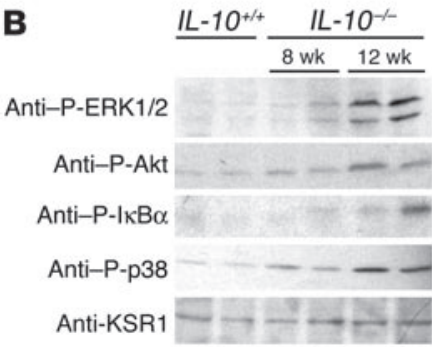

C

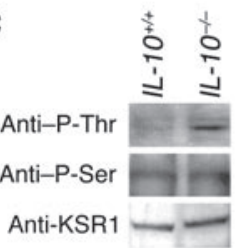

D

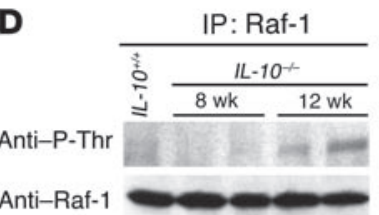

$\mathbf{F}$

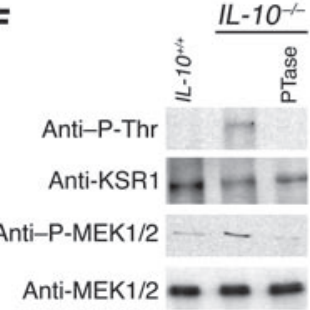

E In vitro kinase assay:

(1) IP KSR1 + Raf-1 + ATP $\downarrow$

(2) KI MEK1 + Raf-1-P + ATP $\downarrow$

\section{Figure 4}

KSR1 is activated in inflamed colon mucosa. (A) H\&E staining of paraffin-embedded colon tissues prepared from $/ L-10^{+/ /}(B A L B / C)$ or IL-10-- mice before developing overt signs of colitis (8-weekold mice) or with rectal prolapse (12-week-old mice) showing characteristic epithelial ulceration (magnification, $\times 20$ ). (B) Colon mucosal lysates were prepared from the respective mice for Western blot analysis with indicated antibodies. KSR1 (C, E, and F) or Raf-1 (D) was immunoprecipitated from colon mucosal lysates using anti-KSR1 or anti-Raf-1 antibodies, respectively, and subjected to Western blot analysis to detect phosphorylation levels using anti-P-Thr or anti-P-Ser antibodies. Using a 2 -stage in vitro kinase assay ( $\mathbf{E}$ and $\mathbf{F})$, immunoprecipitated KSR1 was incubated first with recombinant Raf-1, and then Raf-1 was incubated with KI MEK1 as substrate as detailed in Methods. Phosphorylation of Raf-1 or MEK1 was determined using anti-P-Thr or anti-P-MEK1/2 antibodies, respectively. Immunoprecipitated KSR1 was treated with calf intestinal alkaline phosphatase (PTase) prior to incubation with Raf-1 (F), as indicated. The results shown here are from 2 representatives of 5 mice. WBA, Western blot analysis. cassette, and a kinase role, directly phosphorylating and activating Raf- 1 in this pathway. Evidence has been presented for both functions by a number of different laboratories using different systems $(18,23,30,33-35)$. The function of KSR1 as scaffold protein, kinase, or both appears to be cell and context dependent. For example, in intestinal epithelial cells, TNF activation of Raf-1/MEK/ERK MAPK requires KSR1 kinase activity, but EGF activation of the same pathway is KSR1 kinase-independent (ref. 18 and Figure 3A). However, it is clear that KSR1 expression is required for activation of this signal transduction pathway in other cells and tissues $(23,36)$.

Evidence from mice $(25,26)$, C. elegans $(21,36,37)$, Drosophila (22, $23)$, and cell culture $(12,29,38)$ indicates that KSR1 functions in normal growth, development, and differentiation. Furthermore, 2 recent reports suggest that KSR1 may be necessary for Ras-mediated tumorigenesis $(26,39)$. The present study provides evidence for an additional biological function of KSR1 as a molecular switch regulating the activation of cellular survival pathways. Treatment of $K S R 1^{-/-}$mice with TNF rapidly increases apoptosis in intestinal epithelial cells through failure to stimulate known antiapoptotic pathways, including NF-KB, Akt, and ERK1/2/MAPK (Figure 1 and Figure 2). The block in NF-KB activation shown in our results appears to be primarily epithelial, as TNF-induced NF- $\mathrm{BB}$ nuclear translocation and cIAP2 expression are lost in these cells, yet increased endothelial cell NF-KB activation and endothelin expression remain intact (Figure 6C).
The mechanism of NF- $\mathrm{KB}$ regulation by KSR1 in TNF-treated epithelium is unknown, but a recent in vivo report confirmed our observation, in cell culture studies that increased NF-КB activation is an essential cytokine-mediated intestinal cell survival response $(12,40)$. Activation of MEK1 and NF-KB are regulated by different domains on Raf-1 (41), therefore it is tempting to propose that NF- $\mathrm{BB}$ activation is downstream of Raf- 1 in intestinal epithelial cells. The observation that in vivo Akt/PKB activation by TNF requires KSR1 appears novel, although not completely unexpected, as dominant-negative KSR1 expression blocked TNF-mediated Akt/PKB activation (13). Given the evidence that chronic inflammation enhances tumorigenesis (42) and that Raf- 1 and Akt/PKB have been shown to be effective therapeutic targets in tumor models, it is attractive to speculate that inhibiting KSR1 might be a novel approach to tumor therapy by increasing susceptibility of tumor cells to cytokine-induced apoptosis.

KSR1 Thr phosphorylation state and kinase activity are increased in inflamed $I L-10^{-/-}$mucosa (Figure 4), a disease characterized by an early increase in TNF and IFN- $\gamma(28)$ that is reversed by TNF ablation (43). Neither the mechanism for increased Thr phosphorylation nor the role of phosphorylation in KSR1 kinase activity has been shown. Interestingly, TNF treatment of intestinal epithelial cells increases both Thr and Ser phosphorylation of KSR1 (18). Twelve sites of phosphorylation on KSR1 have been reported, including Ser sites regulated by MEK1/2 and C-TAK- 1 $(29,30,44)$. Dephosphorylation of Ser392 by protein phosphatase 
A
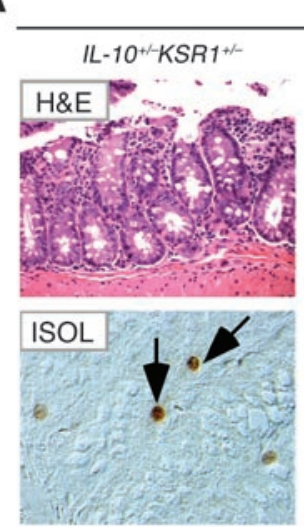

BALB/c $\times 129 /$ SvEv
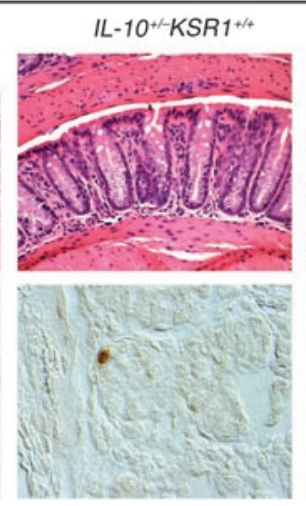

$\mathbf{B}$

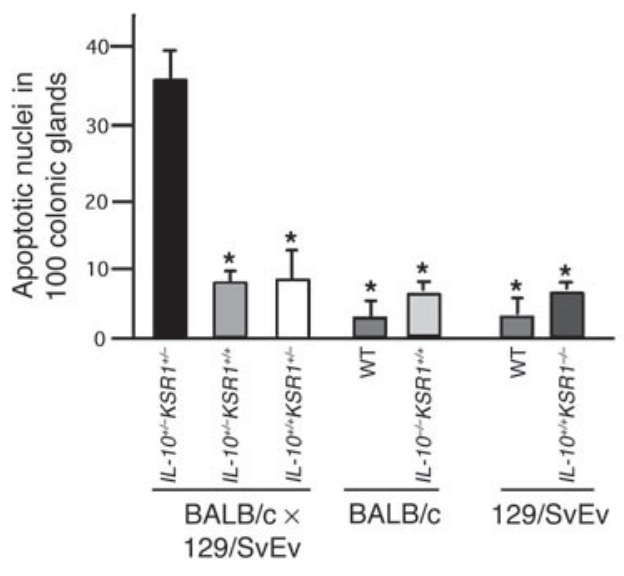

PP2A is a necessary step in KSR1 membrane translocation and Raf-1 activation (45). Our findings show that the phosphatase treatment of KSR1 endogenously phosphorylated on Thr/Ser sites ablates kinase activity (Figure 4F) and suggest that, similar to Raf-1, critical phosphorylation events regulate kinase function. Therefore, it will be important to determine the mechanism and role of phosphorylation on KSR1 kinase activity and to determine whether the lack of increased Ser phosphorylation during inflammation explains part of the increased apoptosis seen in epithelial cells in the $I L-10^{-/-}$model of IBD.

The mechanism of increased injury and epithelial apoptosis in the $\mathrm{KSR}^{+/-} \mathrm{IL}-10^{+/-}$mouse is unclear, but suggests a gene-dosing effect. Similar gene-dosing effects have also been found in the intestinal epithelium with the heterozygous loss of TNF-converting enzyme (TACE/ADAM17) which causes reduced EGF receptor function (46). Similarly, the decreased expression of intestinal KSR1 in the $I L-10^{+/-}$background (Figure 1 and Figure 4) appears to exacerbate either potential cytokine or epithelial regulatory defects mediated by the partial loss of IL-10. We have previously shown that decreased levels of KSR1 generated by an antisense construct increase susceptibility to TNF-mediated apoptosis in intestinal epithelial cells (12). However, in the current studies we did not detect functional alterations in signal transduction pathways or cellular apoptosis of KSR1 or IL-10 haploinsufficient mice. Because alterations in these or other cellular programs likely exist, further studies to understand the potential role of gene dosing in disease pathogenesis are needed. The relevance for understanding the impact of gene dosage effects is emphasized by the recent

\section{Figure 5}

Loss of KSR1 increases colon inflammation. IL-10+/-KSR1+/-, IL-10+/$K S R 1^{+/+}$, or $I L-10^{+/+} K S R 1^{+/-}$mice produced by first-generation crosses of $I L-10^{-/-}$and $K S R 1^{-/-}, I L-10^{-/-}$and 129/SvEv (KSR1 $\left.1^{+/+}\right)$, or BALB/C $\left(I L-10^{+/+}\right)$and $K S R 1^{-/-}$mice, respectively, as well as $/ L-10^{-/-}(B A L B / C)$ and $K S R 1^{-/-}(129 / \mathrm{SvEv})$ mice were sacrificed at 8 weeks of age. (A) H\&E staining examples of mouse colons (magnification, $\times 20$ ), and apoptosis detected using an ISOL kit as in Figure 1 are shown (1 representative of each group, 5 mice per group). Arrows represent ISOL-labeled apoptotic nuclei. Histological injury scores appear in Table 1. (B) The number of apoptotic nuclei found per 100 colonic glands is shown $\left({ }^{\star} P<0.001 \mathrm{com}-\right.$

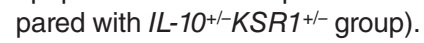

finding that heterozygous carriers of inactivating nucleotide-binding oligomerization domain-2 (NOD2) mutations are at 3-fold increased risk of developing Crohn disease (47).

In summary, this report shows that KSR1 functions in a protective role in the intestinal epithelium exposed to TNF through regulation of antiapoptotic signal transduction pathways. Likewise, increased kinase activity of KSR1 may have a protective role in colon epithelial cells exposed to cytokines during inflammation by promoting intestinal homeostasis, as partial loss of KSR1 expression increases epithelial ulceration and apoptosis. Interestingly,

\section{Table 1}

Colon injury scores for 8-week-old mice

$\begin{array}{lc}\text { Mouse } & \text { Injury score } \\ \text { BALB/c } \times 129 / S v E v & \\ I L-10^{+-} K S R 1^{+/} & 12 \\ I L-10^{+-} K S R 1^{+++} & 2 \\ I L-10^{++} K S R 1^{+/-} & 3 \\ \text { BALB/C } & \\ \text { WT } & 1 \\ I L-10^{-1-K S R 1^{+/+}} & 2 \\ \text { 129/SvEv } & \\ \text { WT } & 2 \\ I L-10^{++} K S R 1^{-/-} & 2\end{array}$

$n=5$ in each group. 
A

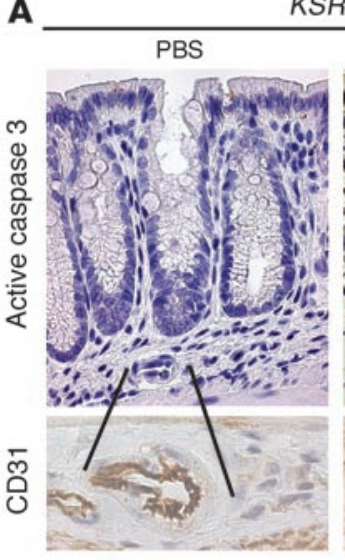

C

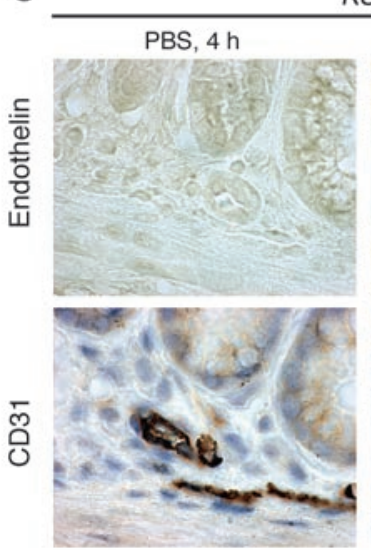

$\mathrm{KSR}^{-1}$

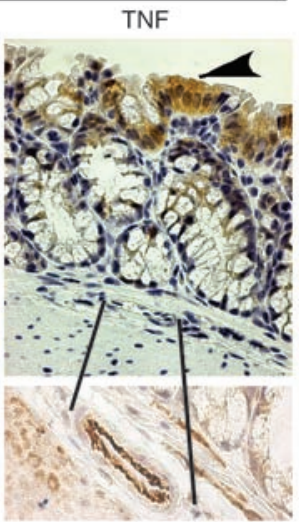

KSR1+/+

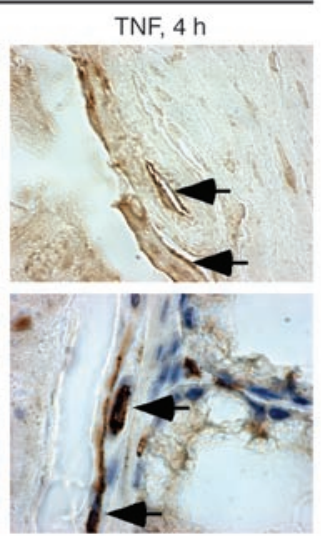

B
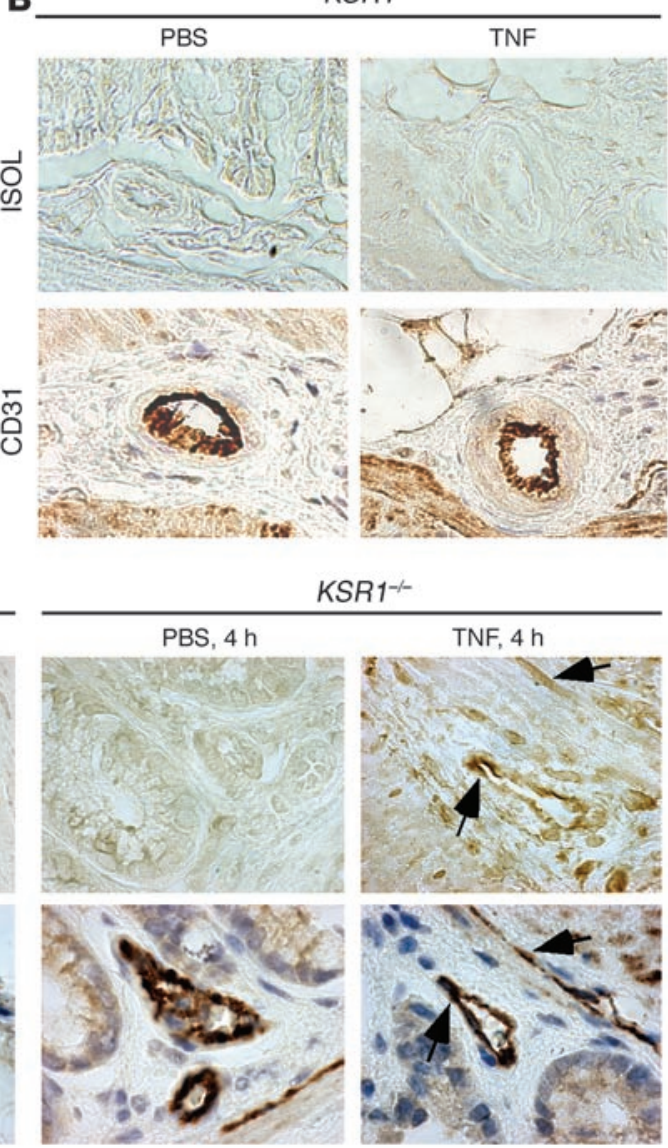

$\mathrm{KSR1}^{-1-}$

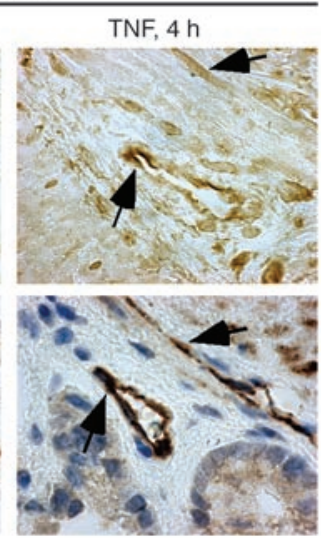

Figure 6

KSR1 is not required for TNF-regulated endothelin expression in intestinal mucosa. Mice were treated with TNF as in Figure 1 and paraffinembedded colon tissues were immunostained with CD31, an endothelial cell marker, active caspase-3 (A), ISOL (B), or the NF-кB-regulated endothelin (C). Positive-staining cells were labeled with peroxidase, developed with 3,3'-diaminobenzidine tetrachloride (DAB) and visualized using DIC microscopy as in Figure 1. CD31, active caspase-3, ISOL, and endothelin immunoassay images were taken from the same areas of adjacent tissue sections. Arrowhead in $\mathbf{A}$ indicates active caspase-3-positive cells. Arrows in $\mathbf{C}$ indicate endothelin- and CD31-labeled cells. Shown is 1 representative of 5 mice for each treatment. Magnification, $\times 40$.

recent reports suggest this cytoprotective role may come at a cost to the organism by supporting Ras-mediated tumorigenesis (26, 39 ). Since patients and mice with chronic IBDs are predisposed to epithelial cancers, the role of KSR1 in this process warrants further investigation. KSR1-deficient mice and colon cell lines will provide new insights into understanding the mechanisms of the epithelial cell responses to inflammation, injury, and repair seen in IBD. These findings also suggest a need for exploration of targeted inhibition of KSR1 to promote epithelial apoptosis in tumorigenesis.

\section{Methods}

Mice and TNF injection. All animal experiments were performed according to protocols approved by the Institutional Animal Care and Use Committee at Vanderbilt University. 129/SvEv and BALB/c background mice were obtained from Taconic Farms and Jackson Laboratories, respectively. KSR1 $1^{-/}$mice on a 129/SvEv background (26) were obtained from Richard Kolesnick (Memorial Sloan-Kettering Cancer Center, New York, New York, USA), and $I L-10^{-/-}$mice on a BALB/c background were obtained from Donna Rennick (DNAX Research Inc.). PCR primers specific for KSR1 and IL-10 transgenes were used for genotyping (sequences available upon request). Male mice (8 weeks old or as indicated, 25-30 g) were anesthetized and then injected i.p. with TNF $\left(10^{4} \mathrm{U}\right.$ in PBS containing $2 \%$ FBS) or with PBS with $2 \%$ FBS alone in a total volume of $200 \mu \mathrm{l}$ for the indicated times.

Cell line preparation, cell culture, and cellular transfection. The young adult mouse colon cells and derivation of conditionally immortalized transgenic cell lines using the $\mathrm{H}-2 \mathrm{~K}^{\mathrm{b}}$-tsA58 mouse (ImmortoMouse; Charles River Laboratories International Inc.) were as previously described $(7,8,27)$. Briefly, $K S R 1^{-/-}$mice were mated with the ImmortoMouse (8). KSR1 $1^{-/-}$mice carrying the heat-labile SV40 gene were sacrificed and colon epithelium was prepared to derive $K S R 1^{-/-}$MCE cell lines as before $(7,8,27)$.

Cell lines were maintained in RPMI 1640 media with 5\% FBS and $5 \mathrm{U} / \mathrm{ml}$ of murine IFN- $\gamma$ on collagen-coated plates and grown under permissive conditions at $33^{\circ} \mathrm{C}$ with $5 \% \mathrm{CO}_{2}$ (7). Before all experiments, cells were transferred to $37^{\circ} \mathrm{C}$ (nonpermissive conditions) with $0.5 \% \mathrm{FBS}$, IFN- $\gamma$-free media overnight. $K S R 1^{--}$MCE cells were transfected with PCMV-Flag-WT KSR1 or pCMV-Flag-KI KSR1 (provided by Richard Kolesnick) using LIPOFECTAMINE reagent (Invitrogen Corp.) as previously described (18).

Tissue and cellular lysate preparations, immunoprecipitation, and Western blot analysis. The colonic mucosa was scraped into homogenization 
buffer and cells were lysed as previously described (48). Cellular lysates were prepared following treatment with $100 \mathrm{ng} / \mathrm{ml}$ murine TNF for 15 minutes (or as indicated) or $10 \mathrm{ng} / \mathrm{ml} \mathrm{EGF}$ (a gift of Stanley Cohen, Vanderbilt University) for 5 minutes. Cells were treated with TNF (100 $\mathrm{ng} / \mathrm{ml}$ for 15 minutes or as indicated) or EGF ( $10 \mathrm{ng} / \mathrm{ml}$ for 5 minutes, gift of Stanley Cohen), and then were scraped into cell lysis buffer as previously described (18).

Immunoprecipitation of KSR1 or Raf-1 from colonic mucosa lysates was performed using monoclonal anti-KSR1 (BD Biosciences) or antiRaf-1 antibody (Upstate USA Inc.). To detect KSR1-interacting proteins in MCE cells, Flag-tagged KSR1 was immunoprecipitated from MCE cells expressing Flag-WT KSR1, Flag-KI KSR1, or vector alone using anti-Flag $\mathrm{M} 2$ affinity gel (Sigma-Aldrich), and washed with $0.3 \mathrm{M} \mathrm{NaCl}$. Flag-KSR1 and its binding proteins were eluted from the gel with $300 \mathrm{ng} / \mu \mathrm{l}$ of $3 \mathrm{xFlag}$ peptide (Sigma-Aldrich), separated on SDS-PAGE, and visualized using the Colloidal Blue staining kit from Invitrogen Corp. Western blot analysis was performed using anti-phospho-Thr anti-phospho-Ser473 Akt antiAkt anti-phospho-Tyr180/182 p38, anti-phospho-Ser32 ІкB $\alpha$, anti-IкB $\alpha$, anti-MEK1/2 (Cell Signaling Technology Inc.), anti-phospho-Thr183/ Tyr185 ERK1/2 MAPK (Promega Corp.), anti-phospho-Ser (P-Ser, Zymed Laboratories Inc.), anti-KSR1, and anti-Raf-1 antibodies.

Histological analysis and immunohistochemistry. Intestinal tissue was immersed in $10 \%$ neutral buffered formalin before sectioning. Paraffinembedded tissue sections were stained with H\&E for light microscopic examination and scoring. Scoring was performed by a single pathologist, blinded to treatment conditions, using an injury scoring system previously described by Kennedy et al. (49), with the total score ranging from 0 (no injury) to 15 (most severe injury).

Slides were immunostained with anti-KSR1, anti-cIAP2 (Santa Cruz Biotechnology Inc.), anti-endothelin (Chemicon International Inc.), anti-CD31 (Santa Cruz Biotechnology Inc.), or anti-active caspase-3 (BD Biosciences) antibodies using reagents provided in the VECTASTAIN $\mathrm{ABC}$ kit (Vector Laboratories Inc.) or the HistoMouse-SP kit (Zymed Laboratories Inc.). Slides were viewed by light or differential interference contrast (DIC) microscopy.

Apoptosis assays. Apoptosis was detected in colon tissue slides with the ApopTag In Situ Oligo Ligation (ISOL) kit (Intergen Co.) using T4 DNA ligase following the manufacturer's guidelines, or by anti-active caspase- 3 staining. Apoptosis in cell lines was determined using ApopTag In Situ Apoptosis Detection kits (TUNEL method, Intergen Co.) and DAPI stain- ing. The slides were observed by DIC or fluorescence microscopy with relative apoptotic cells determined by counting apoptotic cells in at least 100 colonic glands, or by counting 500 cells in culture.

2 -stage in vitro KSR1 kinase assay. We modified our 2 -stage in vitro kinase assay (18) for mouse tissue samples. Immunoprecipitated KSR1 with or without dephosphorylation using $10 \mathrm{U}$ calf intestinal alkaline phosphatase (CIAP, Promega Corp.) was washed with kinase buffer prior to incubation with $2 \mathrm{U}$ recombinant Raf-1 (Upstate USA Inc.) in $30 \mu 1$ kinase buffer with $150 \mu \mathrm{M}$ ATP at $30^{\circ} \mathrm{C}$ for 30 minutes. KSR1 was precipitated by centrifugation, and then the Raf- 1 remaining in the supernatant was incubated with $0.5 \mu \mathrm{g}$ KI MEK1 (Upstate USA Inc.) in $30 \mu \mathrm{l}$ kinase buffer with $150 \mu \mathrm{M}$ ATP at $30^{\circ} \mathrm{C}$ for 30 minutes. MEK1 activation was detected by Western blot analysis with anti-phospho-Ser217/221 MEK1/2 or anti-MEK1/2 (Cell Signaling Technology Inc.) antibodies. Raf- 1 kinase activity in colon mucosal homogenates was determined by incubating immunoprecipitated Raf-1 with KI MEK1 in the kinase buffer. MEK1 activation was detected as described above.

Statistical analysis. The statistical significance of the differences between mean values was determined using paired Student's $t$ test analysis. The level of statistical significance was set at $P<0.05$. The number of apoptotic cells in 100 colonic glands is presented as the mean \pm SD.

\section{Acknowledgments}

We thank Richard Kolesnick (Memorial Sloan-Kettering Cancer Center) for $K S R 1^{-/-}$mice, Robert Whitehead (Vanderbilt University) for helping us to generate the KSR1-/- MCE cell line, Ronald Emeson and Mark Frey (Vanderbilt University) for manuscript suggestions, and the Vanderbilt University Medical Center Imaging Core Research Laboratory (grant CA-68485). This work was supported by NIH grants DK-56008 (to D.B. Polk) and DK-58404 (Vanderbilt University Digestive Disease Research Center).

Received for publication January 8, 2004, and accepted in revised form August 10, 2004.

Address correspondence to: D. Brent Polk, Pediatric Gastroenterology, Hepatology, and Nutrition, S4322 Medical Center North, 21st and Garland Avenues, Nashville, Tennessee 372322576, USA. Phone: (615) 322-7449; Fax: (615) 343-8915; E-mail: d-brent.polk@vanderbilt.edu.
1. Sartor, R.B. 2002. Mucosal immunology and mechanisms of gastrointestinal inflammation. In Sleisenger \& Fordtran's gastrointestinal and liver disease: pathophysiology, diagnosis, management. $\mathrm{M}$. Feldman, L.S. Friedman, and M.H. Sleisenger, editors. W.B. Saunders Co. Philadelphia, Pennsylvania, USA. 21-51.

2. Podolsky, D.K. 2002. Inflammatory bowel disease. N. Engl. J. Med. 347:417-429.

3. Nilsen, E.M., et al. 1998. Gluten induces an intestinal cytokine response strongly dominated by interferon gamma in patients with celiac disease. Gastroenterology. 115:551-563.

4. Schmitz, H., et al. 2002. Supernatants of HIVinfected immune cells affect the barrier function of human HT-29/B6 intestinal epithelial cells. AIDS 16:983-991.

5. Brown, G.R., et al. 1999. Tumor necrosis factor inhibitor ameliorates murine intestinal graft-versus-host disease. Gastroenterology. 116:593-601.

6. Hsueh, W., Caplan, M.S., Tan, X., MacKendrick, W., and Gonzalez-Crussi, F. 1998. Necrotizing enterocolitis of the newborn: pathogenetic concepts in perspective. Pediatr. Dev. Pathol. 1:2-16.

7. Kaiser, G.C., and Polk, D.B. 1997. Tumor necrosis factor $\alpha$ regulates proliferation in a mouse intestinal cell line. Gastroenterology. 112:1231-1240.

8. Corredor, J., et al. 2003. Tumor necrosis factor regulates intestinal epithelial cell migration by receptor-dependent mechanisms. Am. J. Physiol. Cell Physiol. 284:C953-C961.

9. Mizoguchi, E., et al. 2002. Role of tumor necrosis factor receptor 2 (TNFR2) in colonic epithelial hyperplasia and chronic intestinal inflammation in mice. Gastroenterology. 122:134-144.

10. Baker, S.J., and Reddy, E.P. 1996. Transducers of life and death: TNF receptor superfamily and associated proteins. Oncogene. 12:1-9.

11. Warzocha, K., and Salles, G. 1998. The tumor necrosis factor signaling complex: choosing a path toward cell death or cell proliferation. Leuk. Lymphoma. 29:81-92.

12. Yan, F., John, S.K., and Polk, D.B. 2001. Kinase suppressor of Ras determines survival of intestinal cells exposed to tumor necrosis factor. Cancer Res. 61:8668-8675.

13. Yan, F., and Polk, D.B. 2002. Probiotic bacterium prevents cytokine-induced apoptosis in intestinal epithelial cells. J. Biol. Chem. 277:50959-50965.

14. Davis, R.J. 2000. Signal transduction by the JNK group of MAP kinases. Cell. 103:239-252.

15. Xia, Z., Dickens, M., Rainegeaud, J., Davis, R.J., and Greenberg, M.E. 1995. Opposing effects of ERK and JNK-p38 Map kinases on apoptosis. Science. 270:1326-1331.

16. Downward, J. 1998. Ras signalling and apoptosis. Curr. Opin. Genet. Dev. 8:49-54.

17. Troppmair, J., and Rapp, U.R. 2003. Raf and the road to cell survival: a tale of bad spells, ring bearers and detours. Biochem. Pharmacol. 66:1341-1345.

18. Yan, F., and Polk, D.B. 2001. Kinase suppressor of Ras is necessary for tumor necrosis factor $\alpha$ activation of extracellular signal-regulated kinase/ mitogen-activated protein kinase in intestinal epithelial cells. Cancer Res. 61:963-969.

19. Joseph, C.K., Byun, H.-S., Bittman, R., and Kolesnick, R.N. 1993. Substrate recognition by ceramide-activated protein kinase: evidence that kinase activity is proline-directed. J. Biol. Chem. 268:20002-20006.

20. Zhang, Y., et al. 1997. Kinase suppressor of Ras is ceramide-activated protein kinase. Cell. 89:63-72.

21. Kornfeld, K., Hom, D.B., and Horvitz, H.R. 1995. The $k s r-1$ gene encodes a novel protein kinase involved in Ras-mediated signaling in C. elegans. Cell. 83:903-913. 
22. Therrien, M., et al. 1995. KSR, a novel protein kinase required for RAS signal transduction. Cell. 83:879-888.

23. Roy, F., Laberge, G., Douziech, M., Ferland-McCollough, D., and Therrien, M. 2002. KSR is a scaffold required for activation of the ERK/MAPK module. Genes Dev. 16:427-438.

24. Xing, H.R., and Kolesnick, R. 2001. Kinase suppressor of Ras signals through Thr269 of c-Raf-1. J. Biol. Chem. 276:9733-9741.

25. Nguyen, A., et al. 2002. Kinase suppressor of Ras (KSR) is a scaffold which facilitates mitogen-activated protein kinase activation in vivo. Mol. Cell. Biol. 22:3035-3045.

26. Lozano, J., et al. 2003. Deficiency of kinase suppressor of Ras1 prevents oncogenic Ras signaling in mice. Cancer Res. 63:4232-4238.

27. Whitehead, R.H., VanEeden, P.E., Noble, M.D., Ataliotis, P., and Jat, P.S. 1993. Establishment of conditionally immortalized epithelial cell lines from both colon and small intestine of adult $\mathrm{H}-2 \mathrm{~Kb}$-tsA58 transgenic mice. Proc. Natl. Acad. Sci. U. S. A. 90:587-591.

28. Berg, D.J., et al. 1996. Enterocolitis and colon cancer in interleukin-10-deficient mice are associated with aberrant cytokine production and $\mathrm{CD} 4^{+} \mathrm{TH} 1-$ like responses. J. Clin. Invest. 98:1010-1020.

29. Cacace, A.M., et al. 1999. Identification of constitutive and Ras-inducible phosphorylation sites of KSR: implications for 14-3-3 binding, mitogenactivated protein kinase binding, and KSR overexpression. Mol. Cell. Biol. 19:229-240.

30. Muller, J., Ory, S., Copeland, T., Piwnica-Worms, H., and Morrison, D.K. 2001. C-TAK1 regulates Ras signaling by phosphorylating the MAPK scaffold KSR1. Mol. Cell. 8:983-993.

31. Paris, F., et al. 2001. Endothelial apoptosis as the primary lesion initiating intestinal radiation damage in mice. Science. 293:293-297.

32. Ishizuka, T., Takamizawa-Matsumoto, M., Suzuki, K., and Kurita, A. 1999. Endothelin-1 enhances vascular cell adhesion molecule- 1 expression in tumor necrosis factor $\alpha$-stimulated vascular endothelial cells. Eur. J. Pharmacol. 369:237-245.

33. Xing, H.R., Lozano, J., and Kolesnick, R. 2000. Epidermal growth factor treatment enhances the kinase activity of kinase suppressor of Ras. J. Biol. Chem. 275:17276-17280.

34. Wang, X., and Studzinski, G.P. 2004. Kinase suppressor of RAS (KSR) amplifies the differentiation signal provided by low concentrations 1,25-dihydroxyvitamin D3. J. Cell. Physiol. 198:333-342.

35. Yu, W., Fantl, W.J., Harrowe, G., and Williams, L.T. 1997. Regulation of the MAP kinase pathway by mammalian Ksr through direct interaction with MEK and ERK. Curr. Biol. 8:56-64.

36. Ohmachi, M., et al. 2002. C. elegans ksr-1 and ksr-2 have both unique and redundant functions and are required for MPK-1 ERK phosphorylation. Curr. Biol. 12:427-433.

37. Sundaram, M., and Han, M. 1995. The C. elegans $k s r$ - 1 gene encodes a novel Raf-related kinase involved in Ras-mediated signal transduction. Cell. 83:889-901.

38. Muller, J., Cacace, A.M., Lyons, W.E., McGill, C.B., and Morrison, D.K. 2000. Identification of B-KSR1, a novel brain-specific isoform of KSR1 that functions in neuronal signaling. Mol. Cell. Biol. 20:5529-5539.

39. Xing, H.R., et al. 2003. Pharmacologic inactivation of kinase suppressor of ras- 1 abrogates Ras-mediated pancreatic cancer. Nat. Med. 9:1267-1268.

40. Chen, L.W., et al. 2003. The two faces of IKK and
NF-KB inhibition: prevention of systemic inflammation but increased local injury following intestinal ischemia-reperfusion. Nat. Med. 9:575-581.

41. Pearson, G., Bumeister, R., Henry, D.O., Cobb, M.H., and White, M.A. 2000. Uncoupling Raf1 from MEK1/2 impairs only a subset of cellular responses to Raf activation. J. Biol. Chem. 275:37303-37306.

42. van Kempen, L.C., and Coussens, L.M. 2002. MMP9 potentiates pulmonary metastasis formation. Cancer Cell. 2:251-252.

43. Myers, K.J., et al. 2003. Antisense oligonucleotide blockade of tumor necrosis factor-alpha in two murine models of colitis. J. Pharmacol. Exp. Ther. 304:411-424.

44. Volle, D.J., et al. 1999. Phosphorylation of the kinase suppressor of Ras by associated kinases. Biochemistry. 38:5130-5137.

45. Ory, S., Zhou, M., Conrads, T.P., Veenstra, T.D., and Morrison, D.K. 2003. Protein phosphatase 2A positively regulates Ras signaling by dephosphorylating KSR1 and Raf-1 on critical 14-3-3 binding sites. Curr. Biol. 13:1356-1364.

46. Sunnarborg, S.W., et al. 2002. Tumor necrosis factor- $\alpha$ converting enzyme (TACE) regulates epidermal growth factor receptor ligand availability. J. Biol. Chem. 277:12838-12845.

47. Cuthbert, A.P., et al. 2002. The contribution of NOD2 gene mutations to the risk and site of disease in inflammatory bowel disease. Gastroenterology. 122:867-874.

48. Polk, D.B. 1994. Ontogenic regulation of PLC $\gamma 1$ activity and expression in the rat small intestine. Gastroenterology. 107:109-116.

49. Kennedy, R.J., et al. 2000. Interleukin 10-deficient colitis: new similarities to human inflammatory bowel disease. Br. J. Surg. 87:1346-1351. 